\title{
Properties of Random Triangulations and Trees*
}

\author{
L. Devroye, ${ }^{1}$ P. Flajolet, ${ }^{2}$ F. Hurtado, ${ }^{3}$ M. Noy, ${ }^{3}$ and W. Steiger ${ }^{4}$ \\ ${ }^{1}$ School of Computer Science, McGill University, \\ Montreal, Quebec, Canada H3A 2K6 \\ luc@kriek.cs.mcgill.ca \\ ${ }^{2}$ INRIA, \\ Rocquencourt, France \\ Philippe.Flajolet@inria.fr \\ ${ }^{3}$ Universitat Politecnica de Catalunya, \\ Spain \\ \{hurtado,noy\}@ma2.upc.es \\ ${ }^{4}$ Department of Computer Science, Rutgers University, \\ New Brunswick, NJ 08903, USA \\ steiger@cs.rutgers.edu
}

\begin{abstract}
Let $T_{n}$ denote the set of triangulations of a convex polygon $K$ with $n$ sides. We study functions that measure very natural "geometric" features of a triangulation $\tau \in T_{n}$, for example, $\Delta_{n}(\tau)$ which counts the maximal number of diagonals in $\tau$ incident to a single vertex of $K$. It is familiar that $T_{n}$ is bijectively equivalent to $B_{n}$, the set of rooted binary trees with $n-2$ internal nodes, and also to $P_{n}$, the set of nonnegative lattice paths that start at 0 , make $2 n-4$ steps $X_{i}$ of size \pm 1 , and end at $X_{1}+\cdots+X_{2 n-4}=0 . \Delta_{n}$ and the other functions translate into interesting properties of trees in $B_{n}$, and paths in $P_{n}$, that seem not to have been studied before. We treat these functions as random variables under the uniform probability on $T_{n}$ and can describe their behavior quite precisely. A main result is that $\Delta_{n}$ is very close to $\log n$ (all $\operatorname{logs}$ are base 2 ). Finally we describe efficient algorithms to generate triangulations in $T_{n}$ uniformly, and in certain interesting subsets.
\end{abstract}

\footnotetext{
* Part of the work of W. Steiger was supported by a grant from the Generalitat de Catalunya to UPC and part by NSF Grant CCR-9111491. The work of L. Devroye was supported by NSERC Grant A3456. The work of Philippe Flajolet was supported by the L.T.R. Project Alcom-IT (\# 20244) of the European Union.
} 


\section{Introduction and Summary}

Consider a convex polygon $K$ with $n$ sides. We label the vertices $v_{i}=i, i=0, \ldots, n-$ 1 , in clockwise order. A triangulation is a set of $n-3$ noncrossing diagonals $v_{i} v_{j}$ which partitions $K$ into $n-2$ triangles. You can imagine constructing a triangulation $\tau$ recursively: taking the polygon edge $v_{0} v_{n-1}$ as base, just choose the apex of the triangle of $\tau$ that it belongs to, say $v_{i}, 0<i<n-1$, and now continue in the same way on the two polygons $v_{0}, \ldots, v_{i}$ with $v_{0} v_{i}$ as base and $v_{i}, \ldots, v_{n-1}$ with $v_{i} v_{n-1}$ as base (Fig. 1 has $n=8$ and $v_{i}=2,4$, and 5, respectively). This shows that $t_{n}$, the number of such triangulations, satisfies

$$
t_{n}=t_{2} t_{n-1}+t_{3} t_{n-2}+\cdots+t_{n-1} t_{2} \quad t_{2}=t_{3}=1,
$$

the recursion of the Catalan numbers. Therefore

$$
t_{n}=C_{n-2}=\frac{1}{n-1}\left(\begin{array}{c}
2 n-4 \\
n-2
\end{array}\right)
$$

is the size of $T_{n}$, the set of triangulations of $K$.

It is natural to consider certain "geometric" features of a triangulation $\tau \in T_{n}$. Let $d_{i}$ denote the degree of vertex $v_{i}$, the number of diagonals of $\tau$ incident with $v_{i}$. It is easy to see [10] that $\tau$ is characterized by this sequence of degrees. In this paper we study

$$
\Delta_{n}(\tau)=\max \left(d_{i}, i=0, \ldots, n-1\right),
$$

the maximal degree of the vertices. $\Delta_{n}=2$ when $\tau$ is a zigzag and $n-3$ when it is a fan $\left(d_{i}=n-3\right.$ for some vertex $)$, as in Fig. 1 .

Define the length of a diagonal $v_{i} v_{j}$ with $i>j$ to be $\left\|v_{i} v_{j}\right\|=\min (i-j, n-i+j)$, the (fewest) number of successive edges of $K$ between the endpoints. Another geometric feature of $\tau$ that we look at is

$$
\lambda_{n}(\tau)=\max \left(\left\|v_{i} v_{j}\right\|: v_{i} v_{j} \in t\right)
$$

the length of the longest diagonal in the triangulation. It is clear that $n / 3 \leq \lambda_{n} \leq n / 2$.
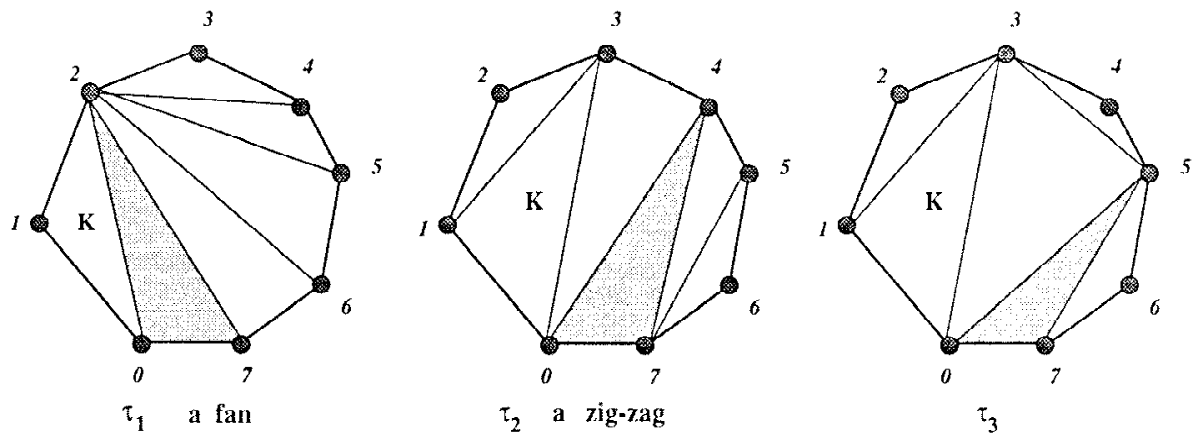

Fig. 1. Three triangulations: $\Delta_{8}=5,2$, and 3, respectively; $\lambda_{8}=4,4$, and 3 , respectively. 
To see how these functions behave across the family of triangulations we treat them as random variables under the uniform probability on $T_{n}$. By symmetry, each $d_{i}$ has the same distribution, but they are not independent because, e.g., $d_{0}+\cdots+d_{n-1}=2(n-3)$. In view of the fact that the expected degree of each $v_{i}$ is $2(1-3 / n)$, it may be somewhat surprising that $\Delta_{n}$ is close to $\log n$ (all $\log$ s are base 2). The main result is

Theorem 1. As $n \rightarrow \infty$,

$$
E\left(\Delta_{n}\right) / \log n \rightarrow 1
$$

In fact $\Delta_{n}$ is strongly concentrated. For all $c>0$, as $n \rightarrow \infty$,

$$
\operatorname{Prob}\left\{\left|\Delta_{n}(\tau)-\log n\right| \leq(1+c) \log \log n\right\} \rightarrow 1 .
$$

The upper bound is based on computing the distribution of $d_{i}$.

Lemma 1. For each vertex $v_{i}$, the probability that its degree is $k$ is given by

$$
\operatorname{Prob}\left(d_{i}=k\right)=\left(\frac{k+1}{2}\right)\left(\frac{n-1}{2 n-5}\right) \prod_{i=1}^{k} \frac{n-2-i}{2 n-5-i} .
$$

Remark 1. Since $\operatorname{Prob}\left(d_{i}=k\right) \leq(k+1) 2^{-(k+1)}$ when $n>3$ (which we assume throughout), this says that $d_{i}$ has tails that decrease geometrically fast. Theorem 1 indicates that their maximum is logarithmic, like the max of $n$ independent geometric random variables (see also Final Remark 1). The proof makes these connections more explicit. It is interesting to wonder about the variance of $\Delta_{n}$. Simulation indicates that it could be constant.

The key fact about the longest diagonal is

Lemma 2. The distribution of the length of the longest diagonal is given by

$$
\operatorname{Prob}\left(\lambda_{n}=k\right)=\frac{n C_{k-1}}{C_{n-2}} \sum_{i=n-k}^{2 k_{(*)}}{ }_{(*)} C_{i-k-1} C_{n-i-1},
$$

where (*) means "multiply the summand by $\frac{1}{2}$ when $i=2 k$ and $i=n-k$, unless $3 k=n$, when we multiply by $\frac{1}{3}$."

This enables us to find the limit distribution of $\lambda_{n}$.

Theorem 2. For each $x \in\left(\frac{1}{3}, \frac{1}{2}\right)$, as $n \rightarrow \infty, \operatorname{Prob}\left(\lambda_{n} \leq n x\right) \rightarrow$ to the distribution with density

$$
w(x)=\frac{1}{\pi} x^{-2}(1-x)^{-2}(3 x-1)(1-2 x)^{-1 / 2} .
$$


In addition $E\left(\lambda_{n}\right) / n \rightarrow \alpha$, where

$$
\begin{aligned}
\alpha & =\frac{\sqrt{3}}{\pi}+\frac{1}{3}-\frac{\log (2+\sqrt{3})}{\pi} \\
& =0.4654615104 \ldots
\end{aligned}
$$

A motivation for the present work-along with deep curiosity about how typical triangulations look-is the inherent interest of binary trees. It is familiar that $T_{n}$ is bijectively equivalent to $B_{n}$, the set of rooted binary trees with $n-2$ internal nodes, each triangulation $\tau \in T_{n}$ corresponding to a particular tree $b(\tau) \in B_{n}$. The two features of triangulations that we study translate into interesting and natural properties of the corresponding trees. For example, $\Delta_{n}(\tau)$ measures a property of $b(\tau)$ that we call the external-node separation, $\chi_{n}(b(\tau))$ : this is the maximal distance in the tree between successive external nodes. $\lambda_{n}(\tau)$ measures a property of $b(\tau)$ that we call the nearly half measure, $H_{n}(b(\tau))$ : it is the size of the largest subtree with not more than half the external nodes. Though trees have been studied intensively (e.g., [3], [4], [8], [11], and [12]), we are unaware of any previous work on these two features. Theorems 1 and 2 and Lemmas 1 and 2 thus appear to express interesting, new facts about trees, as well as about triangulations. In Section 2 we translate the functions $\Delta_{n}$ and $\lambda_{n}$ into the context of binary trees. We also exploit the correspondence between $T_{n}$ and nonnegative lattice paths $P_{n}$; we interpret our functions in this set as well, to help with the proofs, which appear in Section 3.

In Section 4 we describe some linear-time algorithms to generate elements of $T_{n}$ randomly. In addition let $T_{n}(k)$ denote the subset of triangulations in $T_{n}$ with $\lambda_{n}(\tau)=k$. We show how to generate quickly triangulations restricted to $T_{n}(k)$. Finally, if $d_{i}=0$, the vertex $v_{i}$ is called an ear of the triangulation. We show how to generate quickly triangulations with a given number of ears. This may be of some interest because ears of $\tau$ correspond to leaves of $b(\tau)$.

Remark 2. If we regard the trees in $B_{n}$ as binary-search trees generated by permutations of $1, \ldots, n-2$, each permutation being equally likely, the bijection gives the (binary-search tree) probability $\beta$, on $T_{n}$. Trees in $B_{n}$ are well studied in this model (e.g., [4], [6], [11], and [13]). In contrast to the situation in the uniform distribution, the vertex degrees in this model are not identically distributed. Actually $E_{\beta}\left(d_{0}\right)=E_{\beta}\left(d_{n-1}\right)=\Theta(\log n)$ as is familiar from [4] and [6]. We can prove that in this distribution $\Delta_{n} / \log n \rightarrow c>1$ in probability. It seems difficult to analyze $\lambda_{n}$ in this model.

\section{Preliminaries}

We first describe the explicit correspondences between triangulations, trees, and paths that we use. The standard way to associate a tree with a triangulation uses the dual graph of $\tau \in T_{n}$. This gives a binary tree with $n-2$ internal nodes, one for each triangle of $\tau$; adjacent triangles of $\tau$ correspond to nodes joined by an edge of the tree. The triangle with edge $v_{0} v_{n-1}$ is associated with the root of the tree. If $v_{i}$ is the apex of this triangle in $\tau$, label the root with $i$. The left subtree has $i-1$ internal nodes (the number of vertices 

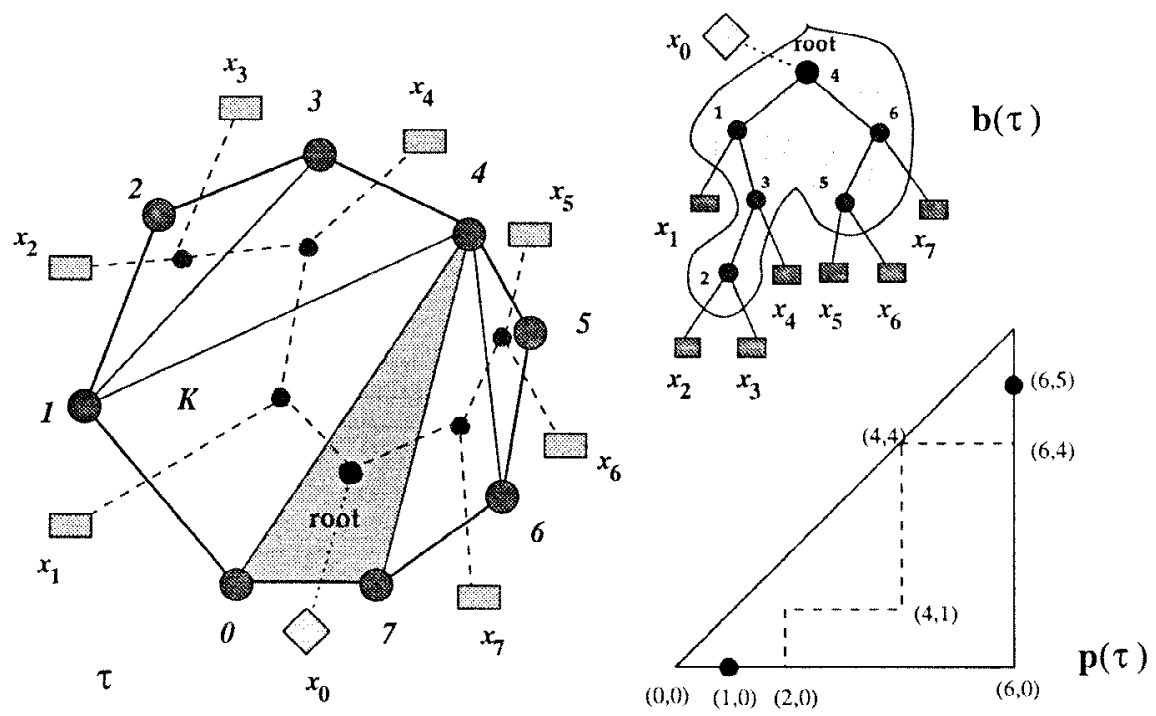

Fig. 2. A triangulation of $K$ and two equivalent representations.

of $K$ between $v_{0}$ and $v_{i}$ ) and corresponds to the triangulation of subpolygon $v_{0}, \ldots, v_{i}$ in $\tau$; the right subtree has $n-2-i$ internal nodes and corresponds to the triangulation of subpolygon $v_{i}, \ldots, v_{n-1}$ in $\tau$, and now continue recursively in the two subpolygons (subtrees). Once the $n-2$ internal nodes are placed, external nodes are added so internal nodes have outdegree 2. Call this (binary-search) tree $b(\tau)$. It has $n-1$ external nodes whose inorder traversal corresponds to the edges $v_{i-1} v_{i}, i=1, \ldots, n-1$. The root misses an external node corresponding to $v_{0} v_{n-1}$. We label them $x_{i}, i=1, \ldots, n-1$, and the missing external node, $x_{0}$ (see Fig. 2). This scheme defines a bijection $T_{n} \leftrightarrow B_{n}$.

For $\tau \in T_{n}$ construct a path $p(\tau) \in P_{n}$ as follows (we think of elements of $P_{n}$ as upright rectilinear paths joining points in the integer lattice in $R^{2}$ contained in the triangle bounded by the $x$-axis, $x=n-2$, and $y=x)$. Paths start at $(1,0)$ and end at $(n-2, n-3)$. Suppose $\tau$ has $j_{0}$ internal diagonals incident to $v_{0}$; then the path moves right $j_{0}$ steps. In general, let $j_{i}$ denote the number of diagonals from $v_{i}$ to a higher number vertex. We are currently at vertex $v_{0}$. We move clockwise in $K$ to the next vertex $v_{i}$ with $j_{i}>0$. The path moves up to the line $y=i$ and then moves right for $j_{i}$ steps. It is easy to see that this procedure gives a path in $P_{n}$ and that every such path comes from a distinct triangulation. These bijections are frequently exploited when studying the combinatorics in one of these sets (see especially [15]), and also for the task of randomly generating elements from one of the sets (e.g., [2], [5], and [13]).

To understand what $\Delta_{n}$ says about trees, imagine the diagonal $v_{i} v_{j}$ in $\tau$ as directed from the smaller numbered vertex of $K$ to the larger one. Take $0<i<n-1$ and move counterclockwise along the circumference of a sufficiently small circle centered at vertex $v_{i}$ from edge $v_{i-1} v_{i}$ to edge $v_{i} v_{i+1}$. First we meet diagonals (if any) coming from lower vertices into $v_{i}$ and then we meet diagonals (if any) going out from $v_{i}$ to higher 
vertices. This shows that the degree of $v_{i}$ in $\tau$ is the number of nodes in $b(\tau)$ between $x_{i}$ and $\alpha$, and the number of nodes between $x_{i+1}$ and $\alpha, \alpha$ being the root of the smallest subtree containing $x_{i}$ and $x_{i+1}$; thus it is the path distance in $b(\tau)$ (number of internal nodes) from $x_{i}$ to $x_{i+1}$, minus 1 . Similarly, and because $x_{0}$ is missing from rooted binary trees, $d_{0}$ and $d_{n-1}$ count the number of internal nodes between the root and $x_{1}$ and the root and $x_{n-1}$, respectively.

Given a rooted binary tree $b$ with $n-2$ internal nodes and external nodes $x_{1}, \ldots, x_{n-1}$, tack an external node $x_{n}\left(\equiv x_{0}\right)$ onto the root and define the external-node separation by

$$
\chi_{n}(b)=\max \left(\left\|x_{i} x_{i+1}\right\|, i=0, \ldots, n-1\right)
$$

where $\left\|x_{i} x_{i+1}\right\|$ counts the path distance minus 1 between the external nodes. We just argued that

Lemma 3. Given $\tau \in T_{n}, \Delta_{n}(\tau)=\chi_{n}(b(\tau))$.

It is more difficult to interpret $\Delta_{n}$ on paths. From the construction of $p(\tau)$ the width $j_{i}$ of the step along $y=i$ is the outdegree of vertex $v_{i}, i=0, \ldots, n-3\left(j_{i}=0\right.$ means the path has no step at level $i$ ). The outdegrees of $v_{n-2}$ and $v_{n-1}$ are zero. Similarly, the indegrees of $v_{0}$ and $v_{1}$ are zero. The other indegrees are more complicated, except for $v_{n-1}$, where the indegree equals $d_{n-1}$, and both count the number of times the path meets $y=x$, from $x=1$ to $x=n-3$. Also both $d_{n-2}$ and the indegree of $v_{n-2}$ can be determined from the intersections of the path with $y=x-1$. However, in general, $d_{i}$ seems not to be an easily "seen" feature of the path.

Given a path $p \in P_{n}$, define its step-width by

$$
s_{n}(p)=\max \left(j_{i}, i=0, \ldots, n-3\right),
$$

where $j_{i}$ is the width of the step of $p$ at height $y=i$. Since $d_{i} \geq j_{i}$,

Lemma 4. Given a triangulation $\tau \in T_{n}, \Delta_{n}(\tau) \geq s_{n}(p(\tau))$.

Therefore probabilistic lower bounds for step-width imply lower bounds for the maximum degree.

It is straightforward to interpret $\lambda_{n}$. From the construction of $b(\tau)$ from $\tau$, each internal node in the tree other than the root corresponds to the part of $\tau$ restricted to some subpolygon $v_{i}, \ldots, v_{j}, i<j-1$. Therefore $\left\|v_{i} v_{j}\right\|$ corresponds to the number of external nodes in the subtree rooted at that particular internal node. Given a tree $b \in B_{n}$, denote its nonroot internal nodes by $v_{i}$ and define $\left\|v_{i}\right\|$ as the number of external nodes in the subtree rooted at $v_{i}$. The "nearly-half measure" of $b$ is defined by

$$
H_{n}(b)=\max \left(\min \left(\left\|v_{i}\right\|, n-\left\|v_{i}\right\|\right), i=1, \ldots, n-3\right) .
$$

Its the size of the largest subtree with not more than half the external nodes. Because $\lambda_{n}(\tau)=H_{n}(b(\tau))$, Lemma 2 gives the distribution of this random variable on trees. 


\section{Proofs}

We sketch the proofs of the results mentioned previously. A main tool is the ballot theorem (see p. 73 of [7]) which says that the number of lattice paths that start at $(0,0)$, make $i$ unit steps to the right, $j \leq i$ unit steps up, and preserve $y \leq x$ is

$$
N_{i j}=\frac{i+1-j}{i+1+j}\left(\begin{array}{c}
i+1+j \\
j
\end{array}\right) .
$$

Proof of Lemma 1. Since the degrees are identically distributed, we only have to consider vertex $v_{0}$. If $d_{0}=k$ in $\tau$, the corresponding path (a good path) must start at $(1,0)$, pass through $(k+1,0)$ and then $(k+1,1)$, and finally continue to $(n-2, n-2)$. The number of ways a path can continue through $(k+1,1)$ to $(n-2, n-2)$ is

$$
N=\frac{k+1}{2 n-5-k}\left(\begin{array}{c}
2 n-5-k \\
n-2
\end{array}\right),
$$

by the ballot theorem. Since there is only one way a path in $P_{n}$ can get from $(1,0)$ to $(k+1,0), N$ is also the number of good paths. Therefore $\operatorname{Prob}\left(d_{0}=k\right)=N / C_{n-2}$. Simplification gives (3).

We prove Theorem 1 in two steps. For the upper bound we want to determine a $k$ $\operatorname{so} \operatorname{Prob}\left(\Delta_{n} \geq k\right) \rightarrow 0$. Observe that $\operatorname{Prob}\left(\Delta_{n} \geq k\right)=\operatorname{Prob}\left(\bigcup_{i=0}^{n-1}\left\{d_{i} \geq k\right\}\right)$ which, by Bonferroni's inequality [7, p. 110] is bounded by $n \operatorname{Prob}\left(d_{0} \geq k\right)$. Lemma 1 shows

$$
\operatorname{Prob}\left(\Delta_{n} \geq k\right) \leq n(k+1) 2^{-k}
$$

which $\rightarrow 0$ for $k \geq \log n+c \log \log n, c>1$.

The lower bound is

Lemma 5. For any $c>0, \operatorname{Prob}\left(\Delta_{n} \leq k\right) \rightarrow 0$ for $k \leq \log n-(1+c) \log \log n$.

Proof. This is the only tricky part, because the $d_{i}$ are dependent. From Lemma $4, \Delta_{n}$ is larger than the size of the largest horizontal step, $s_{n}$, in the corresponding path, so we just need to determine $k$ so that $\operatorname{Prob}\left(\Delta_{n}(\tau) \leq k\right) \leq \operatorname{Prob}\left(s_{n}(p(\tau)) \leq k\right) \rightarrow 0$.

Let $U_{1}, \ldots, U_{2 n-4}$ be a sequence of i.i.d. uniform random variables on $[0,1]$. We describe $\left(X_{m}, Y_{m}\right)$, the coordinates of a point on a random path in $P_{n}$, after $m<2 n-4$ steps of size 1 , each up or right, starting from $(1,0) \equiv\left(X_{0}, Y_{0}\right)$. Of the $C_{n-2}$ paths in $P_{n}, C_{n-3}$ pass through $(1,1)$, the rest through $(2,0)$. Therefore, letting $I_{[A]}$ denote the indicator of $A$, if

$$
\begin{gathered}
X_{1}=I_{\left[U_{1} \leq C_{n-3} / C_{n-2}\right]}, \\
Y_{1}=2-X_{1},
\end{gathered}
$$

$\left(X_{1}, Y_{1}\right)$ will be $(1,1)$ or $(2,0)$ with the correct probabilities. Next suppose $\left(X_{m}, Y_{m}\right)=$ $(i, j)$ is a point on $p$ after $m=i+j-1<2 n-5$ steps from $(1,0)$. By the ballot 
theorem there are

$$
N_{i, j}=\frac{i-j+1}{2 n-3-i-j}\left(\begin{array}{c}
2 n-3-i-j \\
n-1-j
\end{array}\right)
$$

continuations from $(i, j)$ to $(n-2, n-2)$, of which $N_{i+1, j}$ go through $(i+1, j)$. Therefore the probability that the path at $\left(X_{m}, Y_{m}\right)=(i, j)$ moves right at step $m$ is

$$
p_{m}=\frac{N_{i+1, j}}{N_{i, j}}=\left(\frac{i+2-j}{i+1-j}\right)\left(\frac{n-2-i}{2 n-4-i-j}\right),
$$

which is 0 when $i=n-2$, and 1 when $i=j$, as required. If we define

$$
\begin{gathered}
X_{m+1}=X_{m}+I_{\left[U_{m+1} \leq p_{m}\right]}, \\
Y_{m+1}=Y_{m}+\left(1-I_{\left[U_{m+1} \leq p_{m}\right]}\right)
\end{gathered}
$$

our path will move from $(i, j)$ to $\left(X_{m}+1, Y_{m}\right)$ or $\left(X_{m}, Y_{m}+1\right)$ with the correct probabilities. We use $m=i+j-1$ in the equation for $p_{m}$ and simplify to see $p_{m} \geq$

$$
\frac{1}{2}\left(1-\frac{1+m-2 j}{2 n-5-m}\right) \geq \frac{1}{2}\left(1-\frac{1+m}{2 n-5-m}\right) \geq \frac{1}{2}\left(1-\frac{1+m^{*}}{2 n-5-m^{*}}\right),
$$

where $m^{*}>m$ is a bound on the number of steps taken.

Disregarding truncations we define $k=\log n-(1+c) \log \log n, c>0, m^{*}=$ $n /(2 \log n)$, and

$$
p=\frac{1}{2}\left(1-\frac{1}{3 \log n}\right)
$$

With this choice of $m^{*}$ the right-hand side of (10) is at least $p$, if $n$ is large enough.

Consider the Bernoulli sequence $Z_{1}, Z_{2}, \ldots$, where $Z_{i}=I_{\left[U_{i} \leq p\right]}, 1 \leq i \leq m^{*}$, and let $L_{1}, L_{2}, \ldots$ be the lengths of its runs of consecutive ones. Each $Z_{j}=0$ ends such a run and since $I_{\left[U_{j}>p_{j}\right]}$ implies $Z_{j}=0, \Delta_{n} \geq \max L_{i}, i \leq m^{*}$. Therefore

$$
\begin{aligned}
\operatorname{Prob}\left(\Delta_{n}<k\right) & \leq \operatorname{Prob}\left(\bigcap_{i \leq m^{*} / 3}\left(L_{i}<k\right)\right)=\left[\operatorname{Prob}\left(L_{1}<k\right)\right]^{m^{*} / 3} \leq\left(1-p^{k}\right)^{m^{*} / 3} \\
& \leq e^{-p^{k} m^{*} / 3}=e^{-r(\log n)^{c}}
\end{aligned}
$$

for some constant $r>0$. 
Proof of Lemma 2. First observe that there are $C_{k-1} C_{i-k-1} C_{n-i-1}$ triangulations in $T_{n}$ containing $\Delta v_{0} v_{k} v_{i}, i>k$. To count the number of triangulations with $\lambda_{n}=k$, suppose $v_{0} v_{k}$ is a diagonal in $\tau$ (its length is $k$ ) and that $v_{i}$ is the apex of its triangle. If $n-k \leq i \leq 2 k$ neither $\left\|v_{0} v_{i}\right\|$ nor $\left\|v_{i} v_{k}\right\|$ exceeds $k$. So we sum the products $C_{k-1} C_{i-k-1} C_{n-i-1}$ over $i$, from $n-k$ to $2 k$, and multiply this sum by $n$ to reflect the fact that the longest diagonal could as well be $v_{1} v_{k+1}, v_{2} v_{k+2}, \ldots, v_{n-1} v_{k-1}$. Finally the $*$ in $\Sigma_{(*)}$ in (4) means "multiply the summand by $\frac{1}{2}$ when $i=2 k$ and when $i=n-k$ (these triangles have two edges of length $k$ and would be counted twice), unless $3 k=n$, when we multiply by $\frac{1}{3}$." This counts each good triangulation only once.

Proof of Theorem 2. The first observation is that sum (disregarding the meaning of (*)) in (4) has the closed form

$$
\sum_{i=n-k}^{2 k} C_{i-k-1} C_{n-i-1}=\frac{(n-2 k)(3 k+1-n)}{(n-k)(n-k-1)(2 n-4 k-1)}\left(\begin{array}{c}
2 k \\
k
\end{array}\right)\left(\begin{array}{c}
2 n-4 k \\
n-2 k
\end{array}\right),
$$

which can be verified easily. Multiply this sum by $n C_{k-1} / C_{n-2}$, approximate $\left(\begin{array}{c}2 m \\ m\end{array}\right)$ by $4^{m} / \sqrt{\pi m}$, and observe that $w(x)$ is the limit as $k$ and $n \rightarrow \infty$, with $k / n \rightarrow x \in\left(\frac{1}{3}, \frac{1}{2}\right)$. The use of $\Sigma$ in place of $\Sigma_{(*)}$ has no effect on this analysis. The constant $\alpha$ arises from direct evaluation of

$$
\int_{1 / 3}^{1 / 2} x w(x) d x
$$

It is also possible to compute higher moments exactly.

The proofs of the statements in Remark 2 are omitted.

\section{Algorithms}

There already exist algorithms for the uniform generation of elements of $T_{n}, B_{n}$, and $P_{n}$ and which have complexity $O(n)$ in the RAM model of computation. In this section we give a new, extremely simple algorithm, based on the proof of Theorem 1 , to generate elements of $P_{n}$ uniformly. From a random path it is then straightforward to obtain the corresponding trangulation in $T_{n}$ and tree in $B_{n}$ in $O(n)$ time. Using this as a building block we can uniformly generate triangulations with maximum diagonal of a given length and triangulations with a given number of ears, both in linear time. Throughout we use "uniform" to mean "generate a uniform $[0,1]$ random number" and "uniform $[i, i+$ $1, \ldots, j]$ " to mean "generate an integer in $[i, j]$, each being equally likely."

\subsection{Generating Paths}

Given $n$, the following algorithm generates a random path from $(1,0)$ to $(n-2, n-3)$ which is described by $j_{0}, \ldots, j_{n-3}, j_{i}$ giving the width of the step made by the path at level $y=i$, and $j_{0}+\cdots+j_{n-3}=n-3$. 
Algorithm 1. Rand-Path $\left(\mathbf{n} ; \mathbf{j}_{\mathbf{0}}, \ldots, \mathbf{j}_{\mathbf{n}-3}\right)$.

1. (initialize:) $\mathbf{m} \leftarrow \mathbf{0}$; $\left(\mathbf{X}_{\mathrm{m}}, \mathbf{Y}_{\mathrm{m}}\right) \leftarrow(\mathbf{1}, \mathbf{0}) ; \mathbf{i} \leftarrow \mathbf{0} ; \mathbf{j}_{\mathbf{i}} \leftarrow \mathbf{0}$

2. $\mathbf{p}_{\mathbf{m}+\mathbf{1}} \leftarrow\left[\left(\mathbf{X}_{\mathbf{m}}+\mathbf{2}-\mathbf{Y}_{\mathbf{m}}\right)\left(\mathbf{n}-\mathbf{2}-\mathbf{X}_{\mathbf{m}}\right)\right] /\left[\left(\mathbf{X}_{\mathbf{m}}-\mathbf{1}-\mathbf{Y}_{\mathbf{m}}\right)\left(\mathbf{2 n}-\mathbf{4}-\mathbf{X}_{\mathbf{m}}-\mathbf{Y}_{\mathbf{m}}\right)\right]$

3. $\mathbf{U}_{\mathbf{m}+\mathbf{1}} \leftarrow$ uniform; $\mathbf{X}_{\mathbf{m}+\mathbf{1}} \leftarrow \mathbf{X}_{\mathbf{m}}+\mathbf{I}_{\left[\mathbf{U}_{\mathbf{m}+1} \leq \mathbf{p}_{\mathbf{m}+1}\right]}$ $\mathbf{Y}_{\mathbf{m}+\mathbf{1}} \leftarrow \mathbf{Y}_{\mathbf{m}}+\left(\mathbf{1}-\mathbf{I}_{\left[\mathbf{U}_{\mathbf{m}+\mathbf{1}} \leq \mathbf{p}_{\mathbf{m}+\mathbf{1}}\right]}\right)$

4. IF $Y_{m+1}>Y_{m}$ THEN $\left(i \leftarrow i+1 ; j_{i} \leftarrow 0\right)$ ELSE $j_{i} \leftarrow j_{i}+1$

5. $\mathbf{m} \leftarrow \mathbf{m}+\mathbf{1}$; IF $\mathbf{m}<\mathbf{2 n}-\mathbf{5}, \rightarrow \mathbf{2}$.

The algorithm is correct. Step 2, given that $\left(X_{m}, Y_{m}\right)=(i, j)$, computes $p_{m+1}$ as in (9). Therefore $\left(X_{m+1}, Y_{m+1}\right)$ is either $\left(X_{m}+1, Y_{m}\right)$ or $\left(X_{m}, Y_{m}+1\right)$, each with the correct probability. By the remark immediately following (9), this gives a path in $P_{n}$. By induction each path has equal probability.

The algorithm is $O(n)$. Since each $p_{m}$ is computed in constant time, the $2 n-5$ steps take linear time. Since $j_{i}$ is the outdegree of vertex $v_{i}$ in the triangulation $\tau$ that corresponds to the path just generated, it is straightforward now to obtain $\tau$ in linear time, described, for example, by the list of its $n-3$ diagonals. We refer to the process of generating a path and then obtaining its corresponding triangulation by $\operatorname{Rand-Tri}\left(v_{0}, \ldots, v_{n-1}\right)$.

Algorithm 1 is similar to the method of Arnold and Sleep [1] that is mentioned in [5]. A different approach for generating triangulations, paths, or trees appears in [2].

Let $k \geq(n-3) / 2$. Suppose we take $m=0,\left(X_{0}, Y_{0}\right)=(k+1,1), j_{0} \leftarrow k, i \leftarrow 1$, and begin the path algorithm with Step 2, terminating when the path reaches $(n-2, n-3)$. The generated path corresponds to a random triangulation with $\Delta_{n}=k$ and whose maxdegree vertex is $v_{0}$. To randomize the max-degree vertex we choose integer $I$ uniformly in $[0, n-1]$ and $\operatorname{Rotate}(I)$, where $\operatorname{Rotate}(j)$ means "change the vertex labeling of $K$ so $v_{i} \rightarrow v_{(i+j) \bmod n}$. We now have a random triangulation with max-degree $k$, each one being equally likely. It is not clear how to do this efficiently for smaller $k$.

\subsection{Triangulations with Fixed $\lambda$}

Let $T_{n}(k) \subset T_{n}$ denote the triangulations whose longest diagonal has length $k, n / 3 \leq$ $k \leq n / 2$. The proofs of Lemma 2 and Theorem 2 suggest an approach for fast uniform generation of triangulations in $T_{n}(k)$. Suppose that $v_{0} v_{k}$ is the diagonal of max length $(=$ $k$ ) in the desired triangulation. We generate the apex $v_{i}$ of its triangle, with $i \in[n-k, 2 k]$ chosen according to the correct probability. The counting argument used for Lemma 2 shows that $v_{i}$ should be chosen with probability $C_{i-k-1} C_{n-i-1} / S_{2 k}$, where $S_{m}=$ $\sum_{j=n-k}^{m} C_{j-k-1} C_{n-j-1}$. Finally, using Rand-Tri we randomly triangulate the polygons defined by $\left(v_{0}, \ldots, v_{k}\right),\left(v_{k}, \ldots, v_{i}\right)$, and $\left(v_{i}, \ldots, v_{0}\right)$, and then rotate $K$ so diagonal $v_{j} v_{j+k}$ has probability $1 / n$ to be the longest, $j=0, \ldots, n-1$.

Algorithm 2. Random-max-diag $\left(k ; \mathbf{v}_{0}, \ldots, \mathbf{v}_{\mathbf{n}-1}\right)$.

1. $\mathrm{U} \leftarrow$ uniform

2. $\mathbf{i} \leftarrow \min \left(\mathbf{j}: \mathbf{S}_{\mathbf{j}} \geq \mathbf{U} * \mathbf{S}_{\mathbf{2 k}}\right)$

3. Rand-Tri $\left(\mathbf{v}_{0}, \ldots, \mathbf{v}_{k}\right) ; \operatorname{Rand}-\operatorname{Tri}\left(\mathbf{v}_{\mathbf{k}}, \ldots, \mathbf{v}_{\mathbf{i}}\right) ; \operatorname{Rand-Tri}\left(\mathbf{v}_{\mathbf{i}}, \ldots, \mathbf{v}_{\mathbf{0}}\right)$.

4. $\mathbf{I} \leftarrow \operatorname{uniform}(\mathbf{0}, \ldots, \mathbf{n}-\mathbf{1})$

5. Rotate(I) 


\subsection{Triangulations with $k$ Ears}

For $k \in[2,\lfloor n / 2\rfloor]$ let $T_{n}^{k} \subset T_{n}$ denote the triangulations of $K$ with exactly $k$ ears. We give an $O(n)$ algorithm to generate these triangulations uniformly. The algorithm is based on a combinatorial proof of the following formula for the number of $k$-ear triangulations (see [9]):

$$
\left|T_{n}^{k}\right|=\frac{n}{k} 2^{n-2 k}\left(\begin{array}{c}
n-4 \\
n-2 k
\end{array}\right) C_{k-2}
$$

Let $\tau$ be a triangulation of $K$ having ears at vertices $v_{j_{1}}, \ldots, v_{j_{k}}$, and fix $j_{1}=0$. Obviously $\left|j_{i}-j_{i+1}\right| \geq 2$. $\tau$ has $n-3$ diagonals including $v_{j_{i}-1} v_{j_{i}+1}, i=1 \ldots, k$. We collapse $\tau$ by removing (in any order) every edge of $K$ that is not incident to an ear of $\tau, n-2 k$ edges in all. When edge $v_{r} v_{r+1}$ is removed from, say, $v_{r} v_{r+1} v_{q}$, we identify $v_{r+1}$ with $v_{r}$ and note that the two diagonals $v_{q} v_{r}$ and $v_{q} v_{r+1}$ become one, so $n-2 k$ of the diagonals of $\tau$ have also been removed, leaving $2 k-3$.

Let $K^{\prime}$ be the resulting collapsed polygon and $\tau^{\prime}$ its triangulation. Since $K^{\prime}$ is a $2 k-$ gon and $\tau^{\prime}$ has $k$ ears, there are $C_{k-2}$ different possibilities for $\tau^{\prime} ; k$ of its diagonals (one for each ear) form a convex $k$-gon whose interior has $C_{k-2}$ distinct triangulations. To count the number of triangulations $\tau$ that collapse to the same triangulation of $K^{\prime}$, order the $n-3$ diagonals of $\tau$, for example, so $v_{i} v_{j}$ precedes $v_{i} v_{r}$ for diagonals where $i<j<r$ and $v_{i} v_{j}$ precedes $v_{r} v_{s}$ for diagonals where $i<r$ except $d=v_{0} v_{n-1}$ is always last. $d$ remains in $\tau^{\prime}$ but $n-2 k$ of the other $n-4$ are eliminated when $\tau$ collapses to $\tau^{\prime}$ (see Fig. 3). There are $\left(\begin{array}{c}n-4 \\ n-2 k\end{array}\right)$ choices for which diagonals are eliminated, each of which corresponds to a triangulation that collapses to $\tau^{\prime}$. Finally, suppose the diagonal

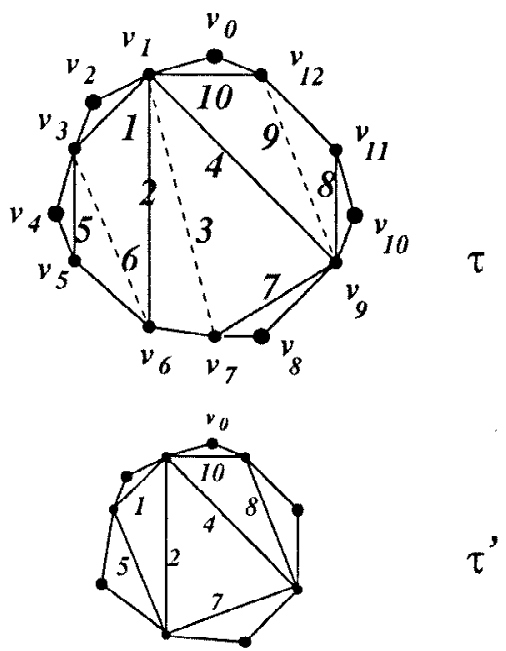

(i)

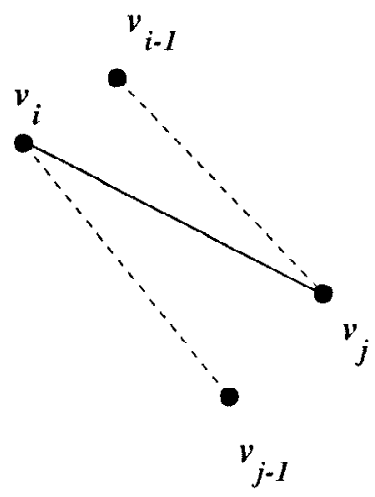

(ii)

Fig. 3. (i) An example of collapse with $n=13$ and $k=5$. The dotted diagonals of $\tau$ disappear in the collapse. (ii) Two distinct ways the predecessor of $v_{i} v_{j}$ could be removed in collapse, or precede $v_{i} v_{j}$ in insertion. 
immediately preceding $v_{i} v_{j}$ in $\tau$ is eliminated in the collapsing. There are two distinct ways this can arise for each eliminated diagonal (see Fig. 3), and thus, $2^{n-2 k}\left(\begin{array}{c}n-4 \\ n-2 k\end{array}\right)$ distinct triangulations $\tau$ collapse to each triangulation $\tau^{\prime}$ of $K^{\prime}$. Finally the term $n / k$ comes from the fact that $v_{0}$ was made to be an ear; every one of the $n$ vertices could play this role but then each triangulation would be counted $k$ times, once for each ear.

This argument underlies the following simple linear-time algorithm to generate a $k$ ear triangulation.

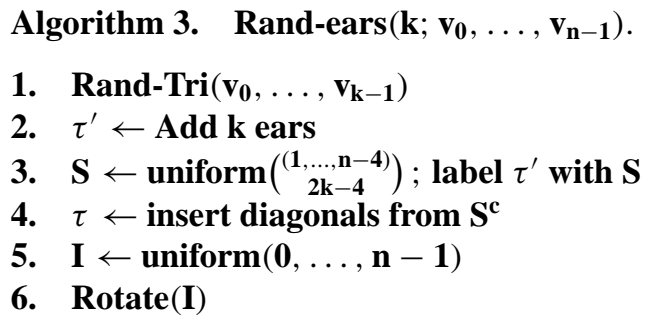

To sketch some details, Step 1 randomly triangulates a $k$-gon. In Step 2 renumber $v_{i} \rightarrow$ $v_{2 i+1}, i=1, \ldots, k$, add vertices $v_{2 i}, i=1, \ldots, k$, and diagonals $v_{2 i-1} v_{2 i+1}, i=$ $1, \ldots, k$; this puts ears at the $k$ even number vertices of $\tau^{\prime}$, the other diagonals being random. In Step 3 diagonal $v_{1} v_{2 k-1}$ is labeled $n-3$, the others are labeled by $1 \leq j_{1}<$ $\cdots<j_{2 k-4} \leq n-4$, the $2 k-4$ elements in $S$, randomly chosen from $1, \ldots, n-4$. Step 4 is done by merging $S^{c}$ into $S \cup(n-3)$. If the current element $r \in S^{c}$ is larger than the current element $s \in S$, we advance to the next element of $S$. Otherwise an edge is inserted into $\tau^{\prime}$ immediately preceding $s=v_{i} v_{j}, i<j$. Specifically, if $U \leftarrow$ uniform is less than 0.5 we create a new vertex between $v_{i-1}$ and $v_{i}$ and the corresponding diagonal to $v_{j}$; otherwise a new vertex appears between $v_{j-1}$ and $v_{j}$ and the corresponding diagonal from $v_{i}$ (see Fig. 3). The details are easily managed in $O(n)$.

\section{Final Remarks}

This paper studied the behavior of two properties of a random triangulation of a convex $n$-gon: (1) $\Delta_{n}$, the maximal degree; (2) $\lambda_{n}$, the length of the longest diagonal. The functions $\Delta_{n}$ and $\lambda_{n}$ correspond to interesting features of binary trees and our results on triangulations give new information about random trees. Some other points are:

1. Following the idea in Remark 1 , as $n \rightarrow \infty$,

$$
\operatorname{Prob}\left(d_{i}=k\right) \rightarrow(k+1) 2^{-(k+2)},
$$

the distribution of $G_{2}$, the sum of two independent geometric $\left(\frac{1}{2}\right)$ random variables. It is interesting to wonder whether $\Delta_{n}$ converges in distribution to the limit distribution of the maximum of $n$ independent copies of $G_{2}$.

2. According to Remark $2, E\left(d_{i}\right)=2(1-3 / n)$ for uniform triangulations but $E_{\beta}\left(d_{0}\right)=E_{\beta}\left(d_{n-1}\right)=\Theta(\log n)$ in search-tree probability, a real difference between the two distributions. At the same time, however, $E\left(\Delta_{n} / \log n\right) \rightarrow 1$ in 
the uniform case and to $c>1$ in the other. For the purpose of comparison, some known properties of random binary trees in the two distributions are:

(a) Height $h_{n}$, the maximal depth of a node. $E\left(h_{n}\right)$ is asymptotic to $2 \sqrt{\pi n}$ in the uniform case [8] and concentrated about $4.31107 \ln n$ in the other [4].

(b) Leaves. In the limit one expects $n / 4$ leaves for uniform trees and $n / 3$ for binary search trees (see [14]).

3. We note that it is easy to generate random triangulations in the search-tree probability. When constructing the triangulation as in the opening paragraph, just choose $v_{i}$ uniformly from $1, \ldots, n-2$, etc. The complexity is $O(n)$.

4. An outstanding question concerns the set $T(K)$ of triangulations of a set $K$ of $n$ points not necessarily in convex position. As opposed to (0), it is not known how to count or even to approximate $|T(K)|$.

\section{Acknowledgments}

We thank Imre Bárány, Alistair Sinclair, and Jean-Marc Fédou for valuable conversations.

\section{References}

1. D. Arnold and M. Sleep. Uniform Random Number Generation of $n$ Balanced Parenthesis Strings. ACM Trans. Prog. Languages Systems 2, 122-128, 1980.

2. M. Atkinson and J. Sack. Generating Binary Trees at Random. Inform. Process. Lett. 41, 21-23, 1992.

3. N. Dershowitz and S. Zaks. Patterns in Trees. Discrete Appl. Math. 25, 241-255, 1989.

4. L. Devroye. A Note on the Height of Binary Search Trees. J. Assoc. Comput. Mach. 33, 489-498, 1986.

5. L. Devroye. Non-Uniform Random Variate Generation, Springer-Verlag, New York, 1986.

6. L. Devroye. Application of the Theory of Records in the Study of Random Trees. Acta Inform. 26, 123-130, 1988.

7. W. Feller. An Introduction to the Theory of Probability and Its Applications, Vol. 1, 3rd Edn., Wiley, New York, 1968.

8. P. Flajolet and A. Odlyzko. The Average Height of Binary Trees and Other Simple Trees. J. Comput. System. Sci. 25, 171-213, 1982.

9. F. Hurtado and M. Noy. Ears of triangulations and Catalan Numbers. Discrete Math. 149, 319-324, 1996.

10. F. Hurtado and M. Noy. The Graph of Triangulations of a Convex Polygon. Proc. Twelfth ACM Symposium on Computational Geometry, N C7-8, 1996.

11. H. Mahmoud. Evolution of Random Search Trees. Wiley, New York, 1992.

12. A. Meir and J. Moon. On the Altitude of Nodes in Random Trees. Canad. J. Math. 30, 997-1015, 1978.

13. B. Pittel. On Growing Random Binary Trees. J. Math. Anal. Appl. 103, 461-480, 1984.

14. R. Sedgewick and P. Flajolet An Introduction to the Analysis of Algorithms. Addison-Wesley, Reading, MA, 1996.

15. D. Sleator, R. Tarjan, and W. Thurston. Rotation Distance, Triangulations, and Hyperbolic Geometry. J. Amer. Math. Soc. 1, 647-681, 1988.

Received August 18, 1997, and in revised form November 5, 1997.

Note added in proof. Gao and Wormald (preprint) recently proved the conjecture in point 1 of the Final Remarks (Section 5) and sharpened the statements in Theorem 1. 